\title{
PRESENT STATUS AND FUTURE PROSPECTS OF LANGMUIR-BLODGETT FILM RESEARCH
}

\section{HANS KUHN}

Max-Planck-Institut für biophysikalische Chemie, Karl-Friedrich-Bonhoeffer-Institut, 3400 Göttingen (F.R.G.)

(Received May 31, 1989; accepted June 7, 1989)

Langmuir-Blodgett (LB) film research has long-term aims (to construct devices for sensing, information processing and chemical processing, as inspired by biosystems) and more direct goals (modelling phenomena in physics, chemistry and biology depending on the precise molecular architecture; and the search for applications in any field where materials with basically new properties which depend on the organization at the molecular level are of interest). Basic to both aspects is the challenge to develop purposely planned supramolecular solid systems (by interlocking appropriate molecules) and a matrix-controlled chemistry (specific reactions driven by the superstructure of interlocking molecules). To indicate ways to approach these goals a number of recent examples are discussed that illustrate the possibilities of constructing purposely designed monolayers and monolayer assemblies.

\section{INTRODUCTION}

The Japanese "sumi nagashi" technique of forming monolayers at a water surface and depositing the film on a solid support was invented about 1000 years ago and is still in use. It is the first example of engineering at the molecular level: Chinese ink, a suspension of carbon particles in a protein solution, is spread on a water surface, and a protein monolayer is formed (as concluded from the area covered after spreading), which is picked up by paper, using the horizontal lifting technique ${ }^{1}$. Agnes Pockels ${ }^{2}, 100$ years ago, realized, when pushing together a soap film at a water surface, that the film pressure increased strongly below a certain area, and Rayleigh ${ }^{3}$ concluded that this is due to the formation of a monomolecular layer and obtained a value for the size of a surfactant molecule, at a time when many scientists did not believe in the reality of atoms. Langmuir and Blodget ${ }^{4}$ studied the detailed structure of fatty acid multilayers obtained by depositing monolayers on a glass slide on top of each other and investigated the detailed conditions for producing such films. George Gaines' famous book ${ }^{5}$ strongly catalyzed further developments.

To see the present status and future prospects of Langmuir-Blodgett (LB) film 
research it is helpful to consider the developments of biology, engineering and chemistry. The paradigm of biology is to consider living objects as organisms, consisting of organs, i.e. purposefully interacting parts, which co-operate and act as functional units. An organism constitutes an entity. Similarly, engineering considers machines as purposefully designed entities of interlocked and interacting parts, functional units, i.e. systems with basically new properties which result from the cooperation of the parts. In contrast, chemistry is concerned with molecules, and the paradigm to learn more about molecules is to focus on pure substances. By this paradigm tremendous development took place, culminating in the synthesis of complex compounds such as vitamins. However, today this paradigm has less driving force. From biology developed molecular biology: biosystems are viewed as supramolecular machines which consist of molecular functional units, i.e. the interacting interlocked parts are single molecules. The challenging prospect is a molecular and supramolecular engineering: the design and fabrication of artificial supramolecular machines. But how should we design a supramolecular machine? Chemists need to synthesize a set of appropriate molecules that interlock to form the machine. The synthesis of different kinds of molecules with the aim of interlocking, to form an entity of purposefully interacting parts should be seen as a new challenge in chemistry: the construction of supramolecular machines by appropriately designing organized molecular aggregates (organizates).

What is the role of LB film research in this challenging new aim of chemistry? Assembling molecules at the air-water interface facilitates interlocking. The fabrication of supramolecular machines is simplified if the molecules can organize themselves at an interface. For that purpose the LB technique has to be extended:

(1) Mixed monolayers: Instead of the fatty acids used in the original LB method a mixture of several kinds of amphiphilic and non-amphiphilic molecules is spread that are functionalized for a given purpose and purposefully designed to interlock to form the intended machinery. In addition, molecules in solution or in the gas phase can bind to the mixed monolayer in a planned manner, thus increasing the complexity of the molecular array. Such a self-organization of a designed assembly taking place at the air-water (gas-liquid) interface is a key feature of engineering at molecular dimensions ${ }^{6}$.

(2) Planned monolayer assembly: Different kinds of designed monolayers are deposited in a planned sequence on top of each other. This is a simple way of purposely arranging molecules such that they form a planned device ${ }^{6,7}$.

(3) Physical manipulation: This can be done in many ways ${ }^{8}$. Monolayer assemblies can be cleaved between distinct monolayers and each part can be printed onto an appropriate surface. An assembly can be put into contact with another assembly with molecular accuracy.

(4) Chemical manipulation: Highly specific reactions can take place driven by the planned superstructure of the designed monolayer assembly (in situ syntheses, in situ polymerizations, in-plane connections, interplane connections ${ }^{6,9,10}$. Chemical manipulaton is crucial for achieving highly complex future machineries.

(5) Controlled adsorption and chemisorption, in situ modification and reaction ${ }^{11}$. The process is technically simple and thus of great interest in applications and in covering large areas with complex molecular assemblies, but it is 
crucially dependent on the reliability of each reaction in producing well-organized molecular arrays.

(6) Assembly modules: Prefabricated supramolecular elements instead of molecules are assembled using LB methods (such as vesicles stabilized by photopolymerization ${ }^{10}$ carrying recognition regions or, in the case of proteins and nucleic acids, entities obtained by gene-technology). This possibility should be of interest in building planned structures at several hierarchical levels. Assembly modules on a two-dimensional (2D) base to form three-dimensional (3D) complex structures should be of interest in future supramolecular engineering strategies.

According to these considerations the challenging new goal in chemistry may be given by the paradigm: synthesize molecules that are tailored to interlock and interact to form a planned solid complex superstructure; use the superstructure as the matrix for chemical reactions-highly specific syntheses or reactions linking discrete parts of superstructure - thus increasing its complexity and intricacy in a planned manner.

\section{GOALS OF CONSTRUCTING PURPOSELY DESIGNED MONOLAYER ASSEMBLIES}

\subsection{Long-term aims}

LB film research is a stimulus in the search for basic attempts at development of sensing and information-processing devices, inspired by biosystems, with molecular functional elements, molecular wiring, supramolecular switching and information processing of signals from moving particles: electrons, protons, solitons, photons. From the present 2D mass data storage one will have to proceed to higher-capacity 3D storage, from 2D signal processing to 3D processing, from information processors based on microlithography to processors that emerge by selforganization at a 2D matrix and develop into $3 \mathrm{D}$ complex arrays ${ }^{12}$.

\subsection{More direct goals: investigation of phenomena and attempted applications}

Investigation of phenomena has two aspects:

(1) To construct simple artificial supramolecular machines in order to learn about possibilities for designing increasingly complex machines, to explore basic mechanisms and materials for a molecular electronics, to develop new methods to fabricate supramolecular devices, to investigate design principles of supramolecular machines, and to demonstrate feasibility as a stimulus to keep on going in a direction in which one cannot expect fast success in attempting important technological changes.

(2) To model systems for research in physics and chemistry in order to investigate processes which depend on the exact molecular architecture, to model bio-functions for understanding bio-processes. Model systems are helpful in many different fields.

Attempted applications can be ${ }^{13}$

(1) to develop new materials (with controlled molecular architecture) for improving traditional technologies: magnetic tape, lubrication, surface coating, etc.;

(2) to develop micro-sensors (pyro-, piezo-, photo-, chemo-sensors);

(3) to develop materials based on co-operativity effects (conduction, photo- 
conduction, magnetism) and switches based on co-operative turnover (supramolecular bistability);

(4) to develop highly selective permeable membranes, surface catalysts for complex reactions, arrangements for solar energy conversion;

(5) to develop materials for integrated optics and optoelectronic devices;

(6) to develop memories with high storage capacity and active "molecular electronic devices" (macroscopic devices based on molecular co-operation, in contrast to the desired true molecular electronic devices considered in Section 2.1.

A given effect depending on the co-operation between single molecules can have many aspects within this frame of reference. This may be illustrated for the energy transfer from a dye molecule that has absorbed a quantum of light $(h v)$ to a second appropriate dye molecule that receives the energy and emits a light quantum $h v^{\prime \prime}$. This co-operation is possible only if the two molecules are at a sufficiently small distance. If the distance is slightly increased the co-operation is lost, and the first molecule emits a quantum $h v^{\prime}$.

The system can be considered

(1) as a prototype of a supramolecular machine (transducing and transmitting signals) which consists of solid parts (the two dye molecules) and mobile parts (the light quanta) ${ }^{\mathbf{1 4}}$;

(2) as a measure for distances in the $20-100 \AA$ range, widely used in developing LB extensions;

(3) as a basic physical process important in understanding photosensitization, as a basic bio-process in light harvesting;

(4) as a tool to study deactivation processes (path of deactivation of excited dye molecules in photochemical reactions and singlet-triplet transitions, nature of dye sensitization of photographic process (electron injection $v$.s. energy transfer) ${ }^{\mathbf{1 5}}$;

(5) as a physical basis for sensors (change in fluorescence color on changing the distance between the two molecules or on changing chemical conditions);

(6) as a prototype of a supramolecular computer if each dye molecule can be switched (by chemical or optical means) from an active state 1 to a passive state 0 and back, thus acting as a logic element.

\section{ORGANIZED MONOLAYERS}

\subsection{Formation of monolayers at air-water interface}

The process of self-organization during film compression can be studied by determining the surface pressure-area isotherm and the surface potential-area isotherm, by measuring the polarized fluorescence and reflection ${ }^{16}$ (which is important to find the orientation of the chromophores at the interface). Recent $\mathrm{X}$-ray and neutron-scattering studies have shown that, after spreading of a C22 fatty acid, islands of tilted molecules are present and that the tilt is gradually diminished when the area is reduced and finally the chains stay vertical. In the case of the cadmium salt the chains are vertical already in the islands. The difference in the surface pressure--area isotherms in the two cases is well explained by this study ${ }^{17}$.

Island formation in spreading a mixed layer makes planned molecular interlocking more difficult. To support transient contacts of molecules the formation 
of $2 \mathrm{D}$ crystals (before the intended interlocking can have taken place) must be avoided. Temporary incorporation of a volatile oil (a molecular lubricant) is essential in complex cases $^{18}$.

\subsection{Designed interlocking}

Steric strain must be avoided, otherwise the interlocking sections cannot meet. This is important in a prefabricated hydrophilic polymer which carries in each monomer unit a hydrocarbon chain bound to the polymer backbone by a hydrophilic intersection ${ }^{19}$. The flexible intersections and the coiled backbone must be sufficiently long to obtain lateral binding and vertical alignment of close-packed hydrocarbon chains, as found from polarized IR studies ${ }^{20}$.

Interlocking sections of component molecules must be exactly planned in designing organized monolayer assemblies. Figure 1 shows some examples in schematic representation. In the first example (Fig. 1(a)) ${ }^{21}$, the molar mixing ratio $1: 2$ of the two compounds with one and two hydrocarbon chains respectively is necessary to obtain a layer which is close packed at the same time in the hydrocarbon part and in the chromophore part. The surface pressure-area isotherm shows a flat part (reflecting the transition from phase 1 to phase 2) followed by a steep rise when the area is reduced (reflecting the close-packed structure in phase 2).

A cyanine dye and an azo-dye are designed to interlock according to Fig. 1(b) ${ }^{22}$. The assembly is formed in a surplus of fatty acid. This can be concluded from surface pressure-area isotherms and polarized light absorption.

Figure $1(\mathrm{c})^{23}$ shows a monolayer of a cyanine dye with two hydrocarbon chain substituents mixed with a long-chain hydrocarbon in 1:1 molar ratio. The hydrocarbon fills the gaps as indicated, and a close-packed layer of hydrocarbon chains is formed; at the same time the chromophores are in a close-packed brickstone arrangement, with the chromophore axes parallel and the chromophore planes perpendicular to the layer plane.

In Fig. 1(d) ${ }^{24}$ the same chromophore as in Fig. 1(c) carries only one long-chain hydrocarbon substituent, and a 1:1 mixture with fatty acid is considered. At sufficiently large areas the fatty acid layer is separated from the dye layer. With decreasing area the fatty acid enters the dye layer and fills the gaps between the hydrocarbon substituents. This is indicated by a flat part in the surface pressure-area isotherm. Above $40 \mathrm{mN} \mathrm{m}^{-1}$ segregation takes place into a phase in which the hydrocarbon chains are close packed and an almost pure dye phase (containing $5 \mathrm{~mol} . \%$ fatty acid). In both phases the chromophores are in a brickstone arrangement, as found from electron diffraction. Thus the fatty acid forms a "solid phase" and a coexisting "vapour phase" within the solid matrix of the dye monolayer.

In Fig. 1(e $)^{25}$ the same chromophore with no long-chain hydrocarbon substituents, dissolved in the subphase, binds to a fatty acid monolayer, again in a brickstone arrangement, as indicated by a strong narrow band in the reflection spectrum. This example illustrates the design of a monolayer by binding a non-amphiphilic compound from the subphase. 

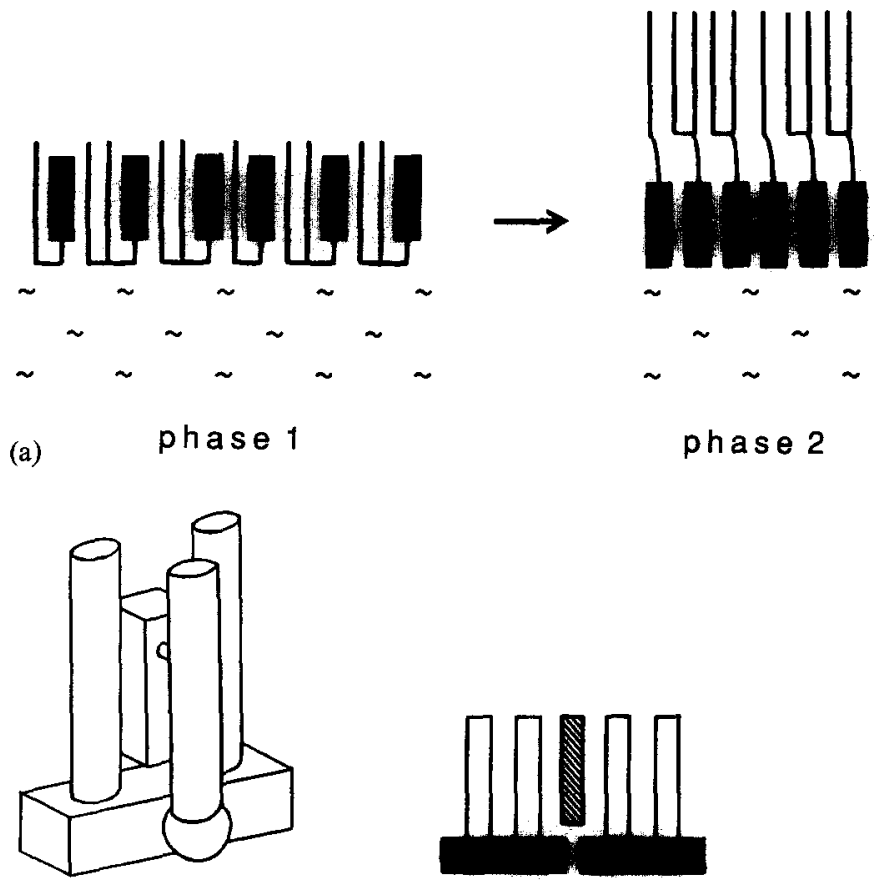

(b)

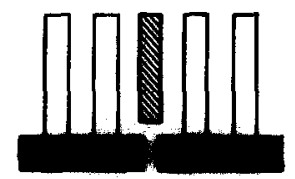

(c)

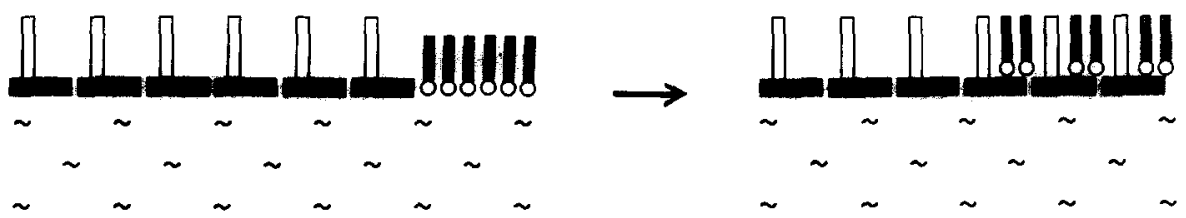

(d)
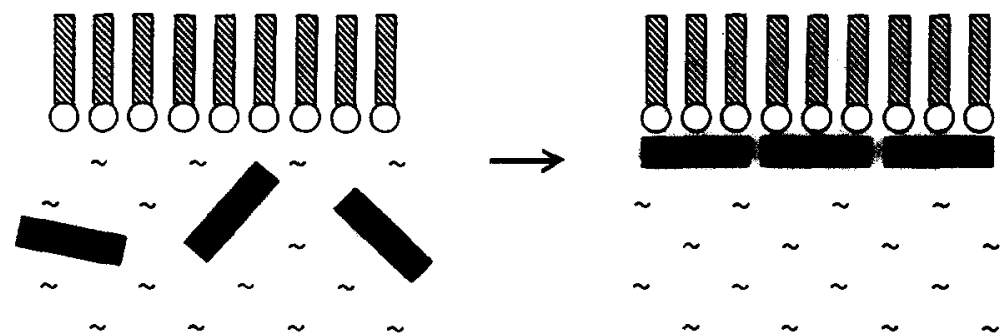

(e)

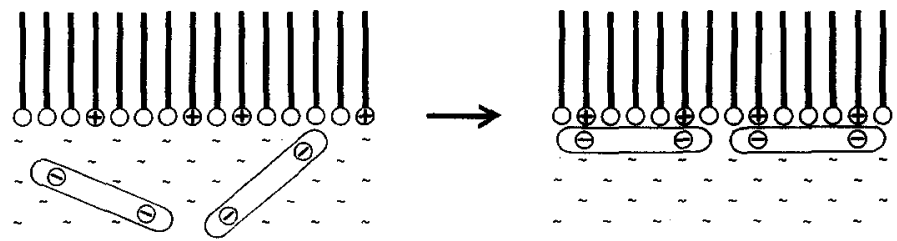

(f)

Fig. 1. Examples illustrating construction of organized monolayers ${ }^{21-26}$. 


\subsection{Adjustment of charges in assembly design}

In Fig. 1(f $)^{26}$ the importance of exact charge adjustment in designing monolayers is indicated. The mixed monolayer of a neutral and a positively charged amphiphile has bound a dye (porphyrin derivative) with four negative charges dissolved in the subphase. A close-packed surfactant monolayer and a compact monolayer of flat dye molecules is obtained only if the ionic charges are well adapted, and this special situation is indicated in the reflection spectrum (which measures the surface coverage of the dye) and in the surface pressure-area isotherm (which shows a steep rise when the area is reduced as the compact arrangement is reached).

If the charge adaptation is not given the dye monolayer can segregate to form 2D micro-crystals of uniform dimension and shape which result from a counterbalance between long-range electrostatic repulsion favouring stretched forms and line tension opposing this ${ }^{27}$. A great variety of shapes of solid domains in fluid monolayers have been observed and studied ${ }^{28}$. Domains can be manipulated by an electrically charged tip near the surface ${ }^{29}$. Such well-defined domains in the micrometer range are of interest for engineering purposes. Design principles have been widely studied in aggregate formation in solution ${ }^{10,30}$.

\subsection{Reactions of molecules in the monolayer with molecules in the subphase}

An amphiphilic cyclodextrine derivative forms good monolayers ${ }^{31}$. Small molecules in the suphase, such as azo-dyes, are bound by entering the hole in cyclodextrine, and an unhampered photoinduced trans to cis and cis to trans conversion takes place. Thus the amphiphile can recognize small molecules and the system with the dye acts as a supramolecular switch.

A monolayer of a porphyrin derivative is of interest because of its co-operative binding and release of four protons. This is concluded from the $\mathrm{pH}$ dependence of the reflection spectrum, which changes completely when the $\mathrm{pH}$ is changed by only 0.2 , from $\mathrm{pH} 0.6$ to $\mathrm{pH} 0.4^{32}$.

Another remarkable co-operative effect is seen in the example of Fig. 1(e) ${ }^{25}$, when another dye is added in much higher dilution to the subphase. The first dye binds to the monolayer as in Fig. 1(e), but after a certain time it is exchanged for the second dye, which is more strongly bound. Each dye forms a brickstone aggregate. No mixed aggregates appear, as seen from the time evolution of the reflection spectrum, which shows that the process is entirely co-operative.

An interesting case of surface recognition is observed if an appropriate monolayer is produced at the surface of a solution of glycine. When the area covered by the film is decreased, a glycine crystal is formed after a certain surface pressure is reached. Thus a specific monolayer matrix must be present to trigger nucleation ${ }^{33}$.

Another example of surface recognition is observed in the case of a monolayer of a biotin lipid at the surface of a solution of a fluorescence-labelled protein with two specific binding sites (streptovidin). Well-shaped 2D crystals are formed. The action of an enzyme (phospholipase A2) can be observed in a monolayer of lecithin containing solid domains in the fluorescence-labelled fluid monolayer. This enzyme hydrolyzes the solid domains, seen in the fluorescence microscope. The domains appear to be gradually eaten up $^{34}$. 


\section{ORGANIZED MONOLAYER ASSEMBLIES}

\subsection{Monolayer deposition}

In monolayer deposition it is important to check transfer ratio and speed. The transfer ratio is unity in usual cases (hydrophilic-hydrophilic and hydrophobic-hydrophobic contact). More critical is the transfer when hydrophobic-hydrophilic contact (with no reorganization taking place) is attempted. If a slide with a hydrophobic surface (covered by a fatty acid monolayer) is taken out of water covered by a monolayer of octadecylamine this layer is not transferred; the transfer ratio is 0 . However, if the fatty acid is exchanged for the fluorinated derivative with a $\mathrm{CF}_{3}$ instead of a $\mathrm{CH}_{3}$ head group, the layer is completely transferred; the transfer ratio is $1^{35}$.

The maximum speed to transfer a layer depends on details in the molecular geometry and charge distribution. For example, a monolayer of arachidic acid is transferred with a maximum speed of $0.5 \mathrm{~cm} \mathrm{~s}^{-1}$ onto a hydrophilic surface by vertical lifting, whereas a monolayer of the corresponding methylester cannot be transferred. For a 1:1 mixture of acid and ester the maximum speed of transfer is highest $\left(1.0 \mathrm{~cm} \mathrm{~s}^{-1}\right)^{36}$.

Molecular orientation can take place during the deposition process depending on the rheological properties of the film and on geometrical conditions. For recent theoretical and experimental studies see ref. 37.

\subsection{Molecular replica formation}

In the arrangement of Fig. 1(f) the negatively charged groups in the dye are compensated by counterions in the mixed monolayer of neutral and positively charged molecules which arrange themselves to form a replica of the pattern of negative charges. When the layer is deposited on a solid support this replica is frozen and the pattern of positive charges is unchanged when the dye is removed. The layer is again exposed to a solution of the dye and the dye is re-adsorbed, in contrast to what is observed when a corresponding monolayer with a frozen statistical charge distribution is exposed ${ }^{38}$. This example shows that artificial formation of a molecular replica and its recognition is possible. The result has implications in the search for sensors, surface-controlled catalysis and for copying information.

Another case to demonstrate the feasibility of copying information at the molecular level is the deposition of a monolayer of a cyanine dye in fatty acid onto another layer of a cyanine dye in fatty acid which has been deposited on a solid support ${ }^{39}$. The chromophores in this layer (first layer) are frozen in their position and orientation and the chromophores in the second layer arrange themselves accordingly by dimer formation with the chromophores in the first layer. The arrangement can be frozen by fixing the second layer to a solid, and the layers can then be separated. Thus information on the molecular arrangement in the first layer is copied and the copy is separated from the original.

An interesting possibility of molecular recognition is created by making holes of molecular size in a monolayer, which are later occupied by molecules fitting in the hole. The holes are made by chemisorbing an appropriate amphiphile in the presence of a non-chemisorbing amphiphile which is later removed, leaving the molecular hole in the monolayer ${ }^{40}$. 


\subsection{Engineered monolayer assembly architecture}

4.3.1 Second harmonic generation ( $S H G$ ) and pyroelectrics

Polar chromophores should be aligned with their transition moment (SHG) or dipole moment (pyroelectrics) preferably perpendicular to the layer plane. However, to obtain well-ordered multilayers the molecules in adjacent layers should stay head-to-head and tail-to-tail. The engineering problem then is to construct appropriate molecules for even- and odd-numbered layers respectively, with chromophore dipoles oriented oppositely relative to the direction from the hydrophilic to the hydrophobic part of the molecule (for examples see ref. ${ }^{41}$ ).

Engineering the environment of a molecule is important. The chains of pentathienyl, in a mixed layer with fatty acid, stay perfectly perpendicular to the layer plane, as indicated by the absorption spectra for $s$ and $p$ polarization ${ }^{42}$. Wellaligned chromophore axes would be important for SHG, but as pentathienyl is apolar the molecule seems useless for that purpose. However, by appropriately engineering the environment, i.e. by incorporating dipoles, this strongly polarizable chromophore can be polarized in one sense or in the opposite sense (for instance, by using fluorinated arachidic acid or octadecylamine respectively) and a corresponding SHG takes place ${ }^{43}$.

In another example, the group carrying the dipole is chemically bound to a strongly polarizable $\pi$-electron system. A monolayer of the compound is not well packed, but by interlocking an active compound which fills the gaps a reasonably well-packed mixed monolayer is obtained with reasonable properties as an SHG material $^{44}$.

The same type of compounds is of interest as pyro-electric material (for thermal IR imaging for night vision). In this case, the engineering problem is somewhat different. The strongly polarizable system is not required and mixing with fatty acid is important to avoid aggregation and to align the dipoles in a well-ordered matrix ${ }^{45}$.

\subsubsection{Laterally conducting layer}

To engineer laterally conducting monolayers one may attempt close-packed stacks of $\pi$-electron systems in a mixed valence state. The requirement is fulfilled for a mixed monolayer of tetracyanoquinodimethane (TCNQ) chemically bound to a hydrocarbon chain and $\mathrm{TCNQ}^{-}$constituting the counterion of a positively charged amphiphile ( $N$-docosylpyridinium) in 1:1 molar mixing ratio ${ }^{9}$. In this case, a rigid close-packed structure of the hydrocarbon chains, important for a well-defined monolayer architecture, a close-packing of TCNQ stacks and the conditions for a mixed valence state are simultaneously given. No flat part in the surface pressure-area isotherm is observed, which indicates the formation of TCNQ, $\mathrm{TCNQ}^{-}$stacks when the area is decreased.

Another engineering strategy to obtain a mixed valence state is complexing TCNQ, chemically bound to a hydrocarbon chain, with tetramethyltetrathiafulvalene $\left(\right.$ TMTTF) ${ }^{46}$. The monolayer was made at the surface of glycerin. High conductivity was observed after spreading and compressing the film; it increased exactly parallel with the surface pressure. To retain the high conductivity after depositing a single monolayer on a solid support seems to be difficult. Better engineering of the sublayer on which the conducting layer is deposited, to avoid lack of co-operativity, is probably crucial. 
In a recently studied case, the high conductivity decreased with increasing temperature above $200 \mathrm{~K}$, indicating a first case of metallic-like conduction in monolayers ${ }^{47}$. Electron spin resonance studies on TCNQ-TMTTF compound monolayers and on monolayers of $N$-docosylpyridinium (TCNQ) ${ }_{2}{ }^{-}$indicate the presence of one-dimensional chains of TCNQ but a high disorder in the stacks, and a strong coupling of TMTTF radical spins and TCNQ radical spins in the compound monolayer ${ }^{48}$.

The field of synthetic metals, semi-conductors and high-temperature superconductors seems to be a particularly challenging future prospect for LB film research. It offers a unique possibility to change the environment of the conducting portion by purposely structuring the amphiphilic portion of the monolayer and the contacting sublayer and in this manner to engineer the conduction mechanism.

\subsection{Planned chemical reactions in planned superstructure}

In the superstructure of a monolayer assembly made of an amphiphile bound to an amino acid the formation of a polypeptide takes place. The secondary structure of the polypeptide thus obtained depends dramatically on the architecture of the assembly. The polypeptide is formed between two monolayers joined head-to-head. If the heads of both monolayers carry the amino acid the polypeptide occurs in the $\alpha$-helical form; if the heads of only one of the two layers carry the amino acid, the $\beta$-form is made. This shows that the conformation of a growing polymer can be controlled by the arrangement of the monomers in the monolayer assembly ${ }^{49}$.

Conducting multilayers made from TCNQ, $\mathrm{TCNQ}^{-}$derivatives were discussed in Section 4.3.2. Another engineering strategy is to build a multilayer of $N$ docosylpyridinium $\mathrm{TCNQ}^{-}$which is then partly oxidized with iodine to produce a mixed valence state. Before oxidation, $\mathrm{TCNQ}^{-}$dimers are present and the multilayer is loosely structured. Oxidation by iodine is accompanied by a fundamental reorganization into a very compact, specific, well-defined structure. The TCNQ, TCNQ ${ }^{-}$units form stacks, and a highly conducting mixed valence state is obtained ${ }^{50}$.

\subsection{Simple supramolecular machines}

4.5.1. Switching and amplifying

In Section 2 energy transfer arrangements were considered as a molecular device for transducing and switching signals by bringing the molecules into an inactive state and back, chemically, say by protonation and deprotonation, or optically ${ }^{51}$. By combining an energy transfer arrangement with a semiconductor the molecular device can be made somewhat more complex ${ }^{51}$. The molecule that has accepted the energy can transfer an electron to the semiconductor. A current flows, which can be triggered by changing the state of the energy donor or of the energy acceptor.

Energy can be transferred from an excited dye molecule to a surface plasmon on a metal film at small distance, or vice versa ${ }^{52}$. This is another possibility that might be used for molecular switching.

The current in a conducting monolayer can be triggered by breaking and restoring co-operativity. This effect can be used for sensing, switching and signal 
amplification ${ }^{53}$. In the arrangement discussed in Section 4.3, octadecylpyridinium used as counterion of (TCNQ) ${ }_{2}{ }^{-}$has been exchanged for an azo-benzene derivative. The current in the conducting monolayer can be switched on and off by using azobenzene as the trigger, which is photoconverted from trans to cis and cis to trans ${ }^{54}$.

Breaking co-operativity in a brickstone aggregate (Section 3.2) offers another possibility for switching and signal amplification. If a small amount of an appropriate dye is incorporated (one molecule among 10000 of the host) and the host is illuminated, the energy absorbed by the host is transferred to the guest and the guest emits light ${ }^{55}$. This effect disappears when the guest is shifted by a few ångströms to the adjacent monolayer. Thus it should be possible to switch on and off the fluorescene of the guest by any slight modification. The system might be useful as a sensor and an efficient amplifier.

\subsubsection{Addressing single molecules}

Addressing a single molecule by light has not been achieved but it may be asked if this is feasible. An aperture of $500-\AA$ diameter has been made. The aperture was illuminated and the light that passed the aperture was observed. Its intensity was strongly changed by changes behind the aperture ${ }^{56}$. Let us consider a monolayer of the aggregate, described above, in which one molecule of the guest is among 10000 or more molecules of the host. This monolayer is fixed close behind the aperture, thus usually none or occasionally one molecule of the guest will be in the range of the aperture. The fluorescence of the guest must then be usually absent, and is present when a guest molecule is in the range of the aperture. Thus it should be possible to address a single molecule of the guest.

Addressing single molecules by electrons is feasible. Tunneling microscope images of fatty acid monolayers show the hexagonal arrangement of the chains ${ }^{57}$. It will be of great interest to apply this technique to planned mixed monolayers, to identify and address particular single molecules. The current flow through the tip of the tunneling microscope (corresponding to $10^{10}$ electrons s $\mathrm{s}^{-1}$ ) is much larger than the expected tunneling current through a fatty acid molecule (see next section), and this needs clarification. Tip touching and deformation of the film, diminishing strongly the distance between tip and conducting support, has been proposed as a possible explanation ${ }^{58}$. It is supported by the fact that typical forces acting on the tip are of the order of $10^{-9} \mathrm{~N}$ (ref. 59), sufficient to push the tip into the layer of flexible fatty acid chains towards the chain ends fixed at the solid support.

\subsubsection{Electron tunneling}

Stilbene was incorporated in a monolayer of fatty acid and paraquat in the adjacent monolayer. Stilbene was photoexcited and the electron transfer to paraquat studied ${ }^{59}$. The distance between electron donor and acceptor was controlled by the length of the hydrocarbon chain bound to stilbene and used as spacer. Electron transfer competes with fluorescence, and the rate of electron transfer is then measured by the degree of flourescence quenching of stilbene. The rate decreases roughly exponentially with the distance. In the case of photoexcited cyanine dye as the donor, and again paraquat as the acceptor, the exponential decrease was demonstrated for fatty acid spacer layers with $14-22$ carbon atoms ${ }^{61}$. This result is explained by assuming that the electron tunnels through the hydrocarbon portion considered as insulating dielectric. The measured rate 
corresponds to a nanosecond as the time for tunneling over a $20-\AA$ distance. In this case, the tunneling electron is at a high energetic level. The extrapolated tunneling rate from the ground state level is many orders of magnitude smaller. This rate can be measured by sand wiching a monolayer between metal electrodes. The current, for a given applied voltage, decreases exponentially with the spacer layer thickness, as shown for fatty acids with 14-23 carbon atoms $\mathrm{s}^{62,63}$. Related to a single arachidic acid chain and to an applied voltage of $40 \mathrm{mV}$, the current is 15 orders of magnitude smaller than that flowing through the tip of the tunneling microscope at the same applied voltage ${ }^{57}$.

It was recently questioned whether even this small current is due to tunneling through the fatty acid monolayer, or through pinholes and grain boundaries. The coherence of electron transfer measurements in very different situations ${ }^{64}$ is a strong argument for tunneling. Deposited and adsorbed films show exactly the same distance dependence of the current ${ }^{62,63}$ (it seems difficult to understand why such different methods should lead to the same pinhole density). Tunneling devices of 1/3-mm diameter behaved uniformly; the current is scattered less than $20 \%{ }^{61}$ (if pinholes were present in the density observed by electro deposition ( $\mathrm{a}$ few per square millimeter ${ }^{65}$, part of the samples would have no pinholes, and part would have one or two pinholes; if the current flowed through the pinholes the scatter would be enormous). The current is $10^{6}$ times larger for a spacer layer with 14 carbon atoms than for a spacer layer with 23 carbon atoms ${ }^{63}$ (if the current flowed through grain boundaries it should be the same in both cases, provided the uncovered area between grains is equal, and there is no reason why this area should be much different in the two cases).

\subsubsection{Vectorial electron transfer}

Let us consider a dye molecule $\mathrm{D}$ that was excited by light. The electron moves to an acceptor $A$ via a conducting element $W$, a molecular wire ${ }^{22}$. The acceptor must be at a sufficiently large distance to avoid back-transfer by tunneling ${ }^{66}$. The molecule that has lost the electron is recovered by accepting an electron from donor $D^{\prime}$. Several attempts were made to approach this challenging supramolecular engineering problems to design a light-induced electron pump ${ }^{22,42,67}$. In a recent approach ${ }^{68}$, a molecule with three functional components $\mathrm{D}^{\prime} \mathrm{D} W$ was synthesized and incorporated into a fatty acid monolayer, and the layer was deposited on a gold film, which acted as acceptor A. The electronic properties of all components are well adapted and the geometry is engineered by connecting the chromophores with hydrocarbon chains of appropriate length. The measured photocurrent is proportional to the number of molecular devices that are active, i.e. where paraquat (component $\mathrm{W}$ ) is in the oxidized state. In the case of a similar molecule but with no donor $\mathrm{D}^{\prime}$, the photocurrent is smaller ${ }^{69}$, showing that $\mathrm{D}^{\prime}$ (ferrocene) is actively contributing. It will be a challenging task to demonstrate that the system really acts as the intended photo-induced battery. Mixed monolayers of the triad and an amphiphile bound to pyrene were investigated, to model photosynthesis. Pyrene served as an antenna, absorbing light and transferring the energy to component $\mathrm{D}$ (perylene), and thus inducing vectorial electron transfer ${ }^{70}$. Attempts to demonstrate photo-induced $\mathrm{CO}_{2}$ reduction were made by investigating monolayers of a nickel complex know to have high catalytic activity for reduction of $\mathrm{CO}_{2}{ }^{71}$. 


\section{OUTLOOK}

As mentioned in Section 2, the construction of purposely designed monolayer assemblies has long-term aims and more direct goals. Basic to both aspects is the aim to develop planned supramolecular solid systems (by interlocking appropriate molecules) and to plan matrix-controlled chemistry (specific reactions driven by the superstructure of interlocking molecules).

Thinking in terms of supramolecular machines should promote future developments. Questions arising are: how can complex molecular devices be constructed, what design principles can be imagined, how can noise and reliability problems in molecular electronic devices be overcome, how can biosystems be understood from the engineering point of view, how can these mechanisms be applied to engineer artificial systems, and how can a 3D complex system, consisting of several hierarchical levels, be self-organized at a 2D matrix?

The question of how the living machinery emerged (usually seen as a chemical problem concerning the nature of the starting material, or as a general physical problem concerning the development of order from chaos) is also a challenging molecular engineering problem ${ }^{72}$ : What is the basic mechanism in the origin of life, in the emergence of the genetic apparatus, what made the evolutionary machinery start and kept it going, and how can solutions of this problem drive thinking in terms of future technologies based on these evolutionary mechanisms?

LB film research is a strong stimulus to propose theoretical models as attempts to answer such questions and to open the way for future attempts in molecular engineering besides its ultimate prospect of engineering entirely new materials and material systems.

\section{REFERENCES}

1 See, e.g. T. Terada, R. Yamamoto and T. Watanabe, Sci. Pap. Inst. Phys. Chem. Res. Jpn., 23 (1934) 173.

2 A. Pockels, Nature, 43 (1891) 437; 46 (1892) 418; 48 (1893) 152.

3 Lord Rayleigh, Philos. Mag., 48 (1899) 321.

4 K. B. Blodgett and I. Langmuir, Phys. Rev., 51 (1937) 964; for a list of papers by Langmuir and Blodgett, see G. L. Gaines, Thin Solid Films, 99 (1983) ix.

5 G. L. Gaines, Jr., Insoluble Monolayers at Liquid-Gas Interfaces, Wiley-Interscience, New York, 1966.

6 D. Möbius (ed.) Langmuir-Blodgett Films 3, in Thin Solid Films, $159(1,2), 160(1,2)(1988)$; A. Barraud (ed.), Langmuir-Blodgett Films and Related Structures, J. Chim. Phys., 85 (1988) 991; M. Sugi, Thin Solid Films, 152 (1987) 305; M. Sugi, in F. L. Carter, R. E. Siatkowsk and H. Wohltjen (eds.), Molecular Electronic Devices, Elsevier, Amsterdam 1988, p.441; L. M. Blinov, Russ. Chem. Rev., 52 (1983) 713; H. Kuhn, D. Möbius and H. Bücher, in A. Weissberger and B. Rossiter (eds.) Physical Methods of Chemistry, Vol. I, Part 3B, Wiley, New York, 1972, p. 577.

7 M. M. Zwick and H. Kuhn, Z. Naturforsch., I7a (1962) 411.

8 O. Inacker, H. Kuhn, D. Möbius and G. Debuch, Z. Phys. Chem. N.F., 101 (1976) 337.

9 A. Ruaudel-Teixier, J. Chim. Phys., 85 (1988) 1067.

10 H. Ringsdorf, B. Schlarb and J. Venzmer, Angew. Chem. Int. Ed. Engl., 27 (1988) 113.

11 J. Sagiv, J. Am. Chem. Soc., 102 (1980) 92; R. Maoz and J. Sagiv, J. Colloid Interface Sci., 100 (1984) 465; J. Gun and J. Sagiv, J. Colloid Interface Sci., 112 (1986) 457.

12 F. L. Carter (ed.), Molecular Electronic Devices II, Marcel Dekker, New York, 1987. 
13 G. G. Roberts, Adv. Phys., 34 (1985) 475; J. D. Swalen, D. L. Allara, J. D. Andrade, E. A. Chandross, S. Garoff, J. Israelachvili, T. J. McCarthy, R. Murray, R. F. Pease, J. F. Rabolt, K. J. Wynne and K. Yu, Langmuir, 3 (1987) 932; A. Barraud, J. Chim. Phys, 85 (1988) 1121.

14 H. Kuhn, Verhandlungen der Schweizerischen Naturforschenden Gesellschaft, 1965, p. 245.

15 R. Steiger, Photogr. Sci. Eng., 28 (1984) 35; R. Steiger, H. Hediger, P. Junod, H. Kuhn and D. Möbius, Photogr. Sci. Eng., 24 (1980) 185; L. v. Szentpaly, D. Möbius and H. Kuhn, J. Chem. Phys., $52(1970) 4618$.

16 M. Orrit, D. Möbius, U. Lehmann and H. Meyer, J. Chem. Phys., 85 (1986) 4966.

17 N. Dent, M. J. Grundy, R. M. Richardson, S. J. Roser, N. B. Mckeown and M. J. Cook, J. Chim. Phys., 85 (1988) 1003.

18 D. Möbius, Ber. Bunsenges. Phys. Chem., 82 (1978) 848.

19 A. Laschewsky, H. Ringsdorf, G. Schmidt and J. Schneider, J. Am. Chem. Soc., 109 (1987) 788.

20 J. F. Rabolt, J. Chim. Phys., 85 (1988) 1027.

21 J. Heesemann, J. Am. Chem. Soc., 102 (1980) 2176.

22 E. E. Polymeropoulos, D. Möbius and H. Kuhn, J. Chem. Phys., 68 (1978) 3918.

23 H. Bücher and H. Kuhn, Chem. Phys. Lett., 6(1970) 183.

24 C. Duschl, D. Kemper, W. Frey, P. Meller, H. Ringsdorf and W. Knoll, J. Chem. Phys., 93 (1989) 4587.

25 U. Lehmann, Thin Solid Films, 160 (1988) 257.

26 H. Grüniger, D. Möbius and H. Meyer, J. Chem. Phys., 79 (1983) 3701; D. Möbius and H. Grüniger, Bioelectrochem. Bioenergetics, 12 (1984) 375.

27 H. Möhwald, S. Kirstein, H. Haas and M. Flörsheimer, J. Chim. Phys., 85 (1988) 1009.

28 H. Möhwald, Thin Solid Films, 159 (1988) 1; Angew. Chem., 100 (1988) 750.

29 A. Miller and H. Möhwald, Europhys. Lett., 2 (1986) 67; A. Miller, W. Knoll and H. Möhwald, Phys. Rev. Lett., 56 (1986) 2633.

30 T. Kunitake, in H. F. Eicke (ed.), Modern Trends of Colloid Science in Chemistry and Biology, Birkhauser, Basel, 1985, p. 34; M. Shimomura and T. Kunitake, Thin Solid Films, 132 (1985) 243.

31 Y. Kawabata, M. Matsumoto, T. Nakamura, M. Tanaka, E. Manda, H. Takahashi, S. Tamura, W. Tagaki, H. Nakahara and K. Fukuda, Thin Solid Films, 159 (1988) 353; H. Niino, A. Yabe, A. Ouchi, M. Tanaka, Y. Kawabata, S. Tamura, T. Miyasaka, W. Tagaki, H. Nakahara and K. Fukuda, Chem. Lett. (1988) 1227.

32 R. Loschek and D. Möbius, J. Chim. Phys., 85 (1988) 1041

33 E. M. Landau, M. Levanon, L. Leiserowitz, M. Lahav and J. Sagiv, Nature, 318 (1985) 353; S. G. Wolf, E. M. Landau, M. Lehav, L. Leiserowitz, M. Deutsch, K. Kjaer and J. Als-Nielsen, Thin Solid Films, 159 (1988) 29.

34 R. Blankenburg, P. Meller, H. Ringsdorf and C. Salesse, J. Biochemistry, 28 (1989) 8214.

D. W. Grainger, A. Reichert, H. Ringsdorf and C. Salasse, FEBS Lett., 252 (1989) 83.

35 V. Vogel and D. Möbius, unpublished.

36 J. G. Petrov, H. Kuhn and D. Möbius, J. Colloid Interface Sci., 73 (1980) 66.

37 M. Minari, K. Ikegami, S. Kuroda, K. Saito, M. Saito, M. Sugi, Solid State Commun., 65 (1988), 1259. M. Sugi, N. Minari, K. Ikegami, S. Kuroda, K. Saito, M. Saito, Thin Solid Films V. Mizrahi, G. I. Stegeman, V. Knoll, Phys. Rev. A, 39 (1989) 3555., 178 (1989) 157.

38 D. Möbius, Z. Phys. Chem. N.F., 154 (1987) 121.

39 D. Möbius and G. Debuch, Ber. Bunsenges. Phys. Chem., 80 (1976) 1158. H. Kuhn, Pure Appl. Chem., $53(1981) 2105$.

40 J. Sagiv., Isr. J. Chem, I8 (1979), 339, 346.

41 I. Ledoux, D. Josse, P. Fremaux, J. P. Piel, G. Post, J. Zyss, T. McLean, R. A. Hann, P. W. Gordon and S. Allen, Thin Solid Films, 160 (1988) 217; G. H. Davies, J. Yarwood, M. C. Petty and C. A. Jones, Thin Solid Films, 159 (1988) 461.

42 U. Schoeler, K. H. Tews and H. Kuhn, J. Chem. Phys., 61 (1974) 5009.

43 D. Möbius and Lifeng Chi, unpublished.

44 T. Richardson, G. G. Roberts, M. E. C. Polywka and S. G. Davies, Thin Solid Films, 160(1980) 231.

45 G. G. Roberts, B. Holcroft, T. Richardson and R. Colbrook, J. Chim. Phys, 85 (1988) 1093.

46 T. Nakamura, F. Takei, M. Matsumoto, M. Tanaka, T. Sekiguchi, E. Manda, Y. Kawabata and G. Saito, Synth. Met., 19 (1987) 681. 
47 T. Nakamura, K. Kojima, M. Matsumoto, H. Tachibana, M. Tanaka, E. Manda and Y. Kawabata, Chem. Lett. (1989) 367.

48 K.Ikegami, S. Kuroda, M.Saito, K.Saito, M.Sugi, Thin SolidFilms, 160(1988) 139; T.Nakamura, M. Matsumoto and K. Kawabata, Phys. Rev. B, 35 (1987) 3667; K. Ikegami, S. Kuroda, M. Sugi, M. Saito, S. Iizima, T. Nakamura, M. Matsumoto, Y. Kawabata and G. Saito, Synth Met., 19 (1987) 669; K. Ikegami, S. Kuroda, K. Saito, M. Saito, M. Sugi, T. Nakamura, M. Matsumoto, Y. kawabata and G. Saito, Thin Solid Films, 179 (1989) 177; K. Ikegami, S. Kuroda, K. Saito, M. Saito, M. Sugi, T. Nakamura, M. Matsumoto, Y. Kawabata and G. Saito, Synth. Met., 27 (1988) B587.

49 K. Fukuda, Y. Shibasaki and H. Nakahara, Thin Solid Films, $99(1983) 87 ; 133(1985) 39 ; 160(1988)$ 43.

50 M. Vandevyver, J. Richard, A. Barraud, A. Ruaudel-Teixier, M. Lequan and R. M. Lequan, $J$. Chem. Phys., 87 (1987) 6754; A. Barraud, Synth. Metals, 19 (1987) 655; B. Belbeoch, M. Rqulliay and M. Tournary, Thin Solid Films, 134 (1985) 89.

51 W. Arden and P. Fromherz, Ber. Bunsenges. Phys. Chem., 82 (1978) 868; J. Electrochem. Soc., 127 (1980) 370; J. Am. Chem. Soc., 102 (1980) 6211; E. E. Polymeropoulos and D. Möbius, Ber. Bunsenges. Phys. Chem., 83 (1979) 1215; D. Möbius, H. Bücher, H. Kuhn and J. Sondermann, Ber. Bunsenges. Phys. Chem., 73 (1969) 845; E. Ando, J. Hibino, T. Hashida and K. Morimoto, Thin Solid Films, 160 (1988) 279.

52 G. Wähling, H. Raether and D. Möbius, Thin Solid Films, 58 (1979) 391; I. Pockrand, A. Brillante and D. Möbius, Chem. Phys. Lett., 69 (1980) 499; J. Chem. Phys., 77 (1982) 6289; W. Knoll, M. R. Philpott, J. D. Swalen and A. Girlando, J. Chem. Phys., 75 (1981) 4795.

53 L. Henrion, G. Derost, A. Ruaudel-Teixier and A. Barraud, Sensors and Actuators, 14 (1988) 251.

54 H. Tachibana, T. Nakamura, M. Matsumoto, H. Komizu, E. Manda, H. Niino, A. Yabe and Y. kawabata, Thin Solid Films, 179 (1989) 207.

55 D. Möbius and H. Kuhn, J. Appl. Phys., 64 (1988) 5138.

56 U. Ch. Fischer, see ref. 12, p. 417; J. Vac. Sci. Technol. B, 3(1), (1985) 386; U. Dürig and D. W. Pohl and F. Rohner, J. Appl. Phys., 59 (1986) 3318; U. Ch. Fischer, U. T. Dürig and D. W. Pohl, Appl. Phys. Lett., 52 (1988) 249.

57 D. P. E. Smith, A. Bryant, C. F. Quate, J. P. Rabe, Ch. Gerber and J. D. Swalen, Proc. Nat. Acad. Sci. U.S.A., 84 (1987) 969.

58 W. Mizutani, M. Shigeno, K. Saito, K. Watanabe, M. Sugi, M. Ono and K. Kajimura, Jpn. J. Appl. Phys., 27 (1988) 1803.

59 O. Marti, personal communication.

60 W. F. Mooney and D. G. Whitten, J. Am. Chem. Soc., 108 (1986) 5712.

61 H. Kuhn, J. Photochem., 10 (1979) 111; D. Möbius, Acc. Chem. Res., 14 (1981) 63; H. Kuhn, Proc. R.A. Welch Foundation 1986, Advances in Electrochemistry, Houston, TX, p. 339.

62 B. Mann and H. Kuhn, J. Appl. Phys., 42 (1971) 4389.

63 J. Sagiv and E. E. Polymeropoulos, J, Chem. Phys., 69(1978) 1836.

64 E. E. Polymeropoulos, J. Appl. Phys., 48 (1977) 2404; Solid State Commun., 28 (1978) 883; M. Sugi, K. Nembach, D. Möbius and H. Kuhn, Solid State Commun., 15 (1974) 1867; M. Sugi, T. Fukui and S. Iizima, Appl. Phys. Lett., 27 (1975) 559; S. lizima and M. Sugi, Appl. Phys. Lett., 28 (1976) 548; M. Sugi, T. Fukui and S. Iizima, Phys. Rev. B 18 (1978) 725; Mol. Cryst. Liq. Cryst., 50 (1979) 183; M. Sugi and S. Iizima, Appl. Phys. Lett., 34 (1979) 290; M. Sugi, M. Saito, T. Fukui and S. Iizima, Thin Solid Films, 99 (1983) 17.

65 I. R. Peterson, J. Chim. Phys., 85 (1988) 997.

66 H. Kuhn, Phys. Rev., A 34 (1986) 3409.

67 E. E. Polymeropoulos, D. Möbius and H. Kuhn, Thin Solid Films, 68 (1980) 173; K. Saito, M. Yoneyama, M. Saito, K. Ikegami, M. Sugi, T. Nakamura, M. Matsumoto and Y. Kawabata, Thin Solid Films, 160 (1988) 133; M. Saito, M. Sugi, T. Fukui and S. lizima, Thin Solid Films, 100 (1983) 117; H. Schreiber, H. Kuhn and H. D. Försterling, Ber. Bunsenges. Phys. Chem., 91 (1987) 798; F. Willig, R. Eichberger, K. Bitterling, W. S. Durfee, W. Stork and M. van der Auweraer, Ber. Bunsenges. Phys. Chem., 91 (1987) 869.

68 M. Fujihira and H. Yamada, Thin Solid Films, 160 (1988) 125.

69 M. Fujihira and M. Sakomura, Thin Solid Films, 179 (1989) 471. 
70 M. Fujihira, M. Sakomura and T. Kamei, Thin Solid Films, 180 (1989) 43.

71 Y. Hirata, K. Suga and M. Fujihira, Thin Solid Films, 179 (1989) 95.

72 H. Kuhn, IBM J. Res. Dev., 32 (1988) 37. 